\title{
La clínica en la hipertensión pulmonar del adulto
}

\section{Diagnostic examination in pulmonary hypertension of the adult}

\author{
René Agustín Flores-Franco, ${ }^{*}$ Daniel Arturo Frías-Fierro*
}

*Hospital General Regional «Dr. Salvador Zubirán Anchondo», Secretaría de Salud. Chihuahua, Chih., México.

\begin{abstract}
RESUMEN. A pesar de los avances tecnológicos para el diagnóstico de la hipertensión pulmonar, la historia clínica y la exploración física aún se consideran fundamentales como método de escrutinio siempre y cuando se dominen los aspectos teóricos, sus fundamentos y sus limitaciones. Con la identificación de los factores de riesgo, la exclusión de aquellas entidades que forman parte del diagnóstico diferencial, una adecuada auscultación y el análisis del conjunto de variables clínicas obtenidas, se provee de importantes pistas que pudieran auxiliar en la detección lo más oportunamente posible de la enfermedad o contribuir en el seguimiento de estos pacientes.
\end{abstract}

Palabras clave: Hipertensión pulmonar, exploración física, insuficiencia cardíaca derecha, ventrículo derecho.

\section{INTRODUCCIÓN}

La hipertensión pulmonar (HP) no es considerada en sí una enfermedad, sino un síndrome en el cual la presión en la circulación pulmonar se encuentra elevada. Con el advenimiento de metodología diagnóstica sofisticada para la detección de la HP y debido a que ésta representa un conjunto de patologías que requieren de mayor objetividad diagnóstica, la utilidad de la clínica pareciera desvanecer y perder importancia. La HP es relativamente frecuente y se estima como el tercer síndrome cardiovascular más común después de la enfermedad coronaria y la hipertensión arterial sistémica.' La HP es común puesto que puede acompañar a casi todas las enfermedades cardíacas y pulmonares. Los recientes avances en el tratamiento han intensificado el interés en la identificación de pacientes afectados en etapas tempranas dentro de su historia natural. La HP se define como una presión media de la arteria pulmonar (PAPm) en

\section{Correspondencia:}

\section{Dr. René Agustín Flores-Franco}

Hospital General Regional

«Dr. Salvador Zubirán Anchondo», SSA. Chihuahua, Chih., México.

Correo electrónico: rflores99@prontomail.com

Recibido: 15-IV-2019, aceptado: 4-VI-2019.
ABSTRACT. Despite technological advances in the diagnosis of pulmonary hypertension, medical history and physical examination are still considered fundamental as screening method provided the theoretical aspects, their foundations and their limitations are mastered. With the identification of the risk factors, the exclusion of those entities that are part of the differential diagnosis, an adequate auscultation and the analysis of the set of clinical variables obtained, provides important clues that could assist in the detection as early as possible of the disease or contribute to the follow-up of these patients.

Keywords: Pulmonary hypertension, physical examination, right-sided heart failure, right ventricle.

reposo de $\geq 20 \mathrm{mmHg}$ y una resistencia vascular pulmonar $(\mathrm{RVP}) \geq 3$ UW determinadas mediante cateterismo cardíaco derecho. ${ }^{2}$ La clasificación de la Organización Mundial de Salud (OMS) divide la HP en cinco grupos según las similitudes en los enfoques terapéuticos y, en cierta medida, los mecanismos fisiopatológicos. Aunque la historia natural varía según la etiología de la enfermedad, la HP es a menudo una enfermedad progresiva caracterizada por un aumento de la RVP y, consecuentemente, una disminución de la función del ventrículo derecho (VD) debido al incremento en su poscarga.

Uno de los principales problemas para el clínico interesado en la HP ha sido el retraso en el diagnóstico ya iniciado el padecimiento. En la práctica clínica, sin embargo, el diagnóstico comienza con una historia clínica detallada y un examen físico, el cual además de su utilidad en el diagnóstico también nos ayuda en determinar su estado dentro de la historia natural de la enfermedad. Todo lo anterior puede resultar ser demasiado complejo ya que además de realizar un interrogatorio y examen físico orientado hacia los factores de riesgo, también deberá dirigirse hacia aquellas patologías que forman parte del diagnóstico diferencial y las comorbilidades que pudieran oscurecer, cambiar la sintomatología o los hallazgos físicos. No olvidemos que el éxito para un diagnóstico correcto depende de manera importante de los factores que influyen en la probabilidad 
clínica, es decir, en identificar a los pacientes con un elevado riesgo. Al comienzo de la enfermedad, los síntomas de la HP son usualmente leves e inespecíficos y conforme la enfermedad progresa el médico requiere poner en práctica una habilidad clínica compleja y en agonía, la auscultación. ${ }^{3}$ Tal vez esta sea la explicación de por qué el clínico con experiencia no necesariamente tiene que seguir algoritmos clínicos diagnósticos para obtener un diagnóstico correcto. En efecto, es bien sabido que no a todos los médicos se les da la misma facilidad para llegar al diagnóstico correcto., ${ }^{4,5}$ Es ese componente artístico de la medicina que aporta el examen físico a los modelos diagnósticos, que caracteriza al buen clínico y lo diferencia del médico inexperto. Un profesional con un oído entrenado probablemente tendría menos dificultad para reconocer el reforzamiento del componente pulmonar del segundo ruido $\left(\mathrm{S}_{2}\right)$ en comparación con alguien con menos experiencia. ${ }^{6}$ De manera similar, un médico que no está acostumbrado a evaluar los sonidos originados del lado derecho del corazón puede que no aprecie con facilidad los sonidos que son más intensos, o sólo perceptibles durante la inspiración, como un cuarto ruido $\left(\mathrm{S}_{4}\right)$ cardíaco derecho. ${ }^{6}$ La auscultación cardíaca es un proceso altamente cognitivo influenciado por factores del paciente, así como también por el sesgo heurístico del profesional, que contribuye a un acuerdo pobre entre observadores. ${ }^{7}$ Por otro lado, la exclusión es un proceso de diagnóstico muy útil sobre todo cuando se conocen a fondo los padecimientos por excluir. ${ }^{8}$ La exclusión camina de la mano con la experiencia. Así sucede con la HP en la que los signos clínicos poseen una mayor especificidad que sensibilidad diagnóstica. ${ }^{9}$ Por tanto, la función más importante de la clínica es llevar a una evaluación adicional bien sustentada y con ello a un diagnóstico definitivo y un tratamiento lo más oportuno posible de esta afección potencialmente fatal.

La etiología de la HP aguda y de la HP crónica (o persistente) es diferente, pero la patogénesis es la misma y sin tratamiento específico la progresión es hacia la insuficiencia ventricular derecha. Con fines didácticos hemos clasificado los hallazgos clínicos en aquellos presentados durante un evento de HP aguda y diferenciarlos de aquellos presentados en la HP crónica. En cada una de estas dos situaciones, la sintomatología y hallazgos físicos a su vez son clasificados en aquellos relacionados o asociados a la HP per se y aquellos ocasionados por la disfunción del VD. Se acepta que los síntomas y signos son sugestivos, pero inespecíficos, que requieren de estudios confirmatorios y la información clínica puede ser modificada por la presencia de comorbilidades o enfermedades de base como sucede con los pacientes con otras afecciones cardiopulmonares. De hecho, la información disponible proviene de estudios clínicos donde por lo regular no se incluyeron pacientes con comorbilidades. Ya desde 1881, Graham Steell había hecho la observación de que nunca había que confiar en la presencia de los signos físicos en forma aislada para el diagnóstico de la insuficiencia cardíaca y él recomendaba mejor un buen interrogatorio..$^{10}$

\section{Hipertensión pulmonar aguda}

La HP aguda se caracteriza por un aumento repentino de la presión arterial pulmonar. Básicamente es representada por la tromboembolia pulmonar (TEP) aguda; no obstante, también se observa en la lesión pulmonar aguda/síndrome de insuficiencia respiratoria del adulto y en estados de sepsis donde, además de formar parte de la fisiopatología de dichos síndromes representa un marcador de mal pronóstico. ${ }^{11}$

En relación con la TEP la no especificidad de la sintomatología ha hecho necesaria la creación de modelos clínicos de predicción siendo el de Wells el más utilizado; y en el cual la combinación de algunos síntomas y signos incrementan la probabilidad diagnóstica más que su interpretación en forma individual. ${ }^{12}$ Aunque un número cada vez mayor de pruebas diagnósticas están disponibles para el diagnóstico correcto de la TEP, la valoración clínica tiene un papel fundamental dentro de las estrategias diagnósticas. No obstante, la sintomatología no necesariamente está relacionada con el grado de HP y sí lo esté en parte por otros factores, tales como la hiperactividad simpática, atelectasias y disminución de la excursión diafragmática. Así por ejemplo, la disnea, tos, el dolor pleurítico y la hemoptisis, las cuales integran el síndrome de infarto pulmonar, pueden presentarse con una PAPm incluso normal y es debido a la obstrucción de una pequeña arteria pulmonar segmentaria distal. ${ }^{13}$ Aquí, la disnea puede originarse en forma aguda (70\%), en reposo (60\%), manifestarse con el ejercicio $(16 \%)$, como ortopnea (36\%), pero también estar ausente (30\%). ${ }^{14}$ En un inicio no se asocia con algún grado de hipoxemia, pero posiblemente sea debido a la hiperventilación y es importante distinguirla de la disnea que se presenta en la TEP con compromiso hemodinámico. En el estudio PIOPED II, el dolor pleurítico fue ocho veces más frecuente que la hemoptisis y ésta rara vez fue significativa. ${ }^{14}$ Dicho dolor puede asociarse a un frote pleural inspiratorio y espiratorio o estertores finos inspiratorios producidos en la zona del infarto y no modificables con la tos. El dolor puede irradiarse hacia el hemiabdomen superior y confundir al clínico con patología quirúrgica abdominal como la colecistitis. Otros síntomas inespecíficos referidos en este tipo de tromboembolia son la fiebre y con ella la taquicardia. Las sibilancias y una disminución en los ruidos respiratorios también han sido descritos. De existir derrame pleural este sería muy pequeño, exudativo, claro o hemorrágico y prácticamente indetectable por la clínica ocupando en la mayoría de los casos menos del 15\% del hemitórax, bloqueando sólo el seno costodiafragmático en la telerradiografía de tórax. ${ }^{15}$ 
La fuente embolígena en las extremidades inferiores sólo es localizable clínicamente en menos del 30\% de los pacientes y se asume que es debido a que el trombo ya fue desprendido del sistema venoso profundo. ${ }^{16}$

La presencia de múltiples émbolos de pequeño a moderado tamaño durante un período de semanas define al síndrome de tromboembolismo masivo subagudo o de disnea aislada. Debido a que la obstrucción arterial pulmonar por los trombos ocurre paulatinamente, existe el tiempo suficiente para la adaptación del VD ante la elevación de la presión de la arteria pulmonar incrementando su contractilidad y ya con algún grado de hipertrofia concéntrica. Esta elevación de la presión arterial pulmonar también obedece a fenómenos neurohumorales vasoactivos y no sólo a la presencia del trombo. Aquí sí, los síntomas y hallazgos clínicos están relacionados con el incremento de la PAPm que por lo general no sobrepasa los $35 \mathrm{mmHg}{ }^{17} \mathrm{La}$ disnea se hace más evidente sobre todo durante el ejercicio, pero sin una taquicardia proporcional al grado de disnea o a alteraciones en la presión arterial sistémica y es debido a que el gasto cardíaco es bien mantenido. ${ }^{17}$ Tampoco otros síntomas de alarma como aquellos asociados al infarto pulmonar están presentes o de presentarse éstos serían intermitentes. El grado de obstrucción de la circulación pulmonar no supera aún el 50\%, pero además de los signos sugestivos de infarto(s) pulmonar(es), lo habitual es que haya hipoxemia y cianosis central. ${ }^{17}$ Puede también haber dolor tipo anginoso, palpitaciones y anormalidades electrocardiográficas. Debido al carácter repetitivo del tromboembolismo es más probable encontrar evidencia de trombosis venosa profunda (TVP) en alguna de las extremidades inferiores, neoplasias o algún factor desencadenante, habitualmente la inmovilización por motivos no quirúrgicos. ${ }^{18}$ Asimismo, en pacientes con TVP distal a la vena poplítea se ha documentado una prevalencia del $13 \%$ de TEP submasiva silenciosa. ${ }^{19} \mathrm{~A}$ la auscultación ya puede ser evidente con ayuda del diafragma del estetoscopio, el reforzamiento del componente pulmonar con o sin desdoblamiento de $\mathrm{S}_{2}$ y con ayuda de la campana del estetoscopio escuchar un cuarto ruido cardíaco $\left(\mathrm{S}_{4}\right)$, todos ellos mejor audibles en la región del borde esternal izquierdo, a la altura del tercero al quinto espacio intercostal y en la región subxifoidea o epigástrica, de preferencia con el paciente en decúbito dorsal. Esta última zona de auscultación es donde se proyecta el VD y, por lo tanto, se auscultan mejor los ruidos anormales provenientes del él, además de que en pacientes con enfermedad pulmonar obstructiva crónica (EPOC), donde el enfisema hace muy pobres las ventanas acústicas cardiovasculares convencionales, la auscultación en el epigastrio pudiera ser una buena opción (figura 1). ${ }^{20}$ En condiciones normales, mientras que el componente aórtico de $\mathrm{S}_{2}\left(\mathrm{~S}_{2 \mathrm{a}}\right)$ se escucha en los cuatro focos cardíacos de auscultación, el componente pulmonar $\left(\mathrm{S}_{2 \mathrm{p}}\right)$ sólo es auscultable en el foco pulmonar y ocasionalmente en el ápex.
La transmisión de $S_{2 p}$ fuera del foco pulmonar usualmente significa la presencia de HP. ${ }^{21}$ Existe un reforzamiento de $\mathrm{S}_{2 \mathrm{p}}$ cuando éste es más intenso en el foco pulmonar que en el foco aórtico, siempre y cuando no exista hipotensión arterial sistémica que debilite relativamente a $\mathrm{S}_{2 \mathrm{a}}$. No obstante, en la TEP subaguda masiva la PAPm no es tan elevada como sucede en la HP crónica, como para hacer tan evidente este signo. Además, deberá considerarse que la intensidad de los sonidos cardíacos precordiales está influenciada por factores anatómicos como el índice de masa corporal, las características de la caja torácica y el eje cardíaco. Sin embargo, el clínico deberá esforzarse en la búsqueda de dichos signos.

Por un lado, la HP ocasiona los cambios auscultatorios descritos en $\mathrm{S}_{2}$ y por otro lado, la hipertrofia en sí hace al ventrículo más rígido ocasionando un $\mathrm{S}_{4}$ derecho durante la fase de llenado ventricular. En la primera situación, y como será visto más adelante en la sección de HP crónica, existe un retardo en el cierre de las valvas pulmonares debido a que la sístole ventricular derecha es prolongada por la $\mathrm{HP}^{22}$ El desdoblamiento del $\mathrm{S}_{2}$ no es tan amplio como en la HP crónica y sólo se presenta durante la inspiración. A diferencia del desdoblamiento fisiológico de $\mathrm{S}_{2}$, el cual se presenta por lo general en niños y adolescentes y se ausculta mejor en el ápice cardíaco, en la HP el componente pulmonar es más acentuado. Asimismo, un $\mathrm{S}_{4}$ derecho deberá distinguirse del $\mathrm{S}_{4}$ del adulto mayor producido por la rigidez ventricular propia de la cardioangioesclerosis senil e hipertensión arterial sistémica la cual normalmente es audible en el ápice cardíaco y no es más evidente durante la inspiración como sucede con un $\mathrm{S}_{4}$ derecho. La presencia de un $\mathrm{S}_{4}$ traduce hipertrofia ventricular y una

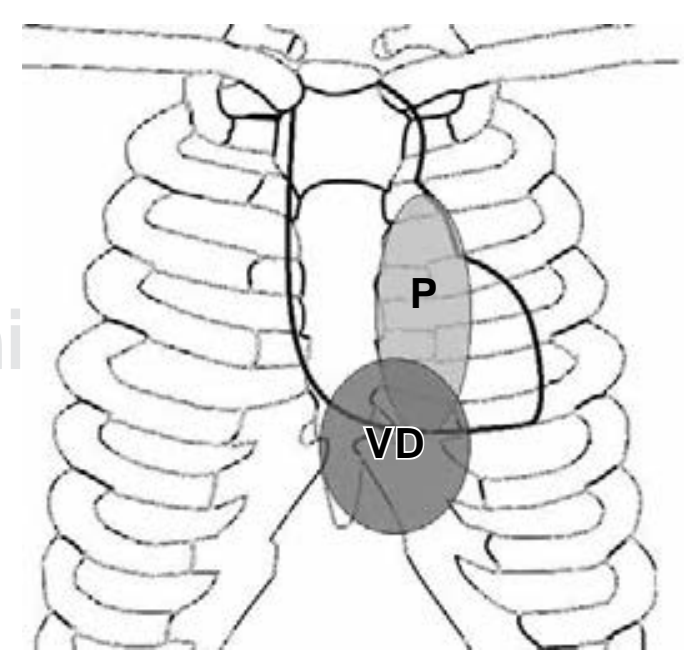

Figura 1: Zonas de auscultación cardíaca derecha en relación a la caja torácica y el esternón.

$\mathrm{P}=$ Zona de la pulmonar; $\mathrm{VD}=$ Zona del ventrículo derecho (modificado de Hill NS). ${ }^{20}$ 
PAPm significativamente elevada, quizás ya con cierto grado de cronicidad y suele irradiarse hacia las venas yugulares.

También se ha descrito la presencia, durante la auscultación, de soplos en la región interescapulovertebral producidos por la obstrucción parcial de las arterias pulmonares por el trombo. ${ }^{23,24}$ Pueden ser sistólicos o continuos, más aparentes durante una inspiración profunda y desaparecer con el tratamiento anticoagulante o persistir como sucede en la TEP crónica en la que, además, se han atribuido a la circulación bronquial colateral. Finalmente, es cosa de tiempo y de que la TEP subaguda no tratada evolucione a una TEP crónica, o bien, se presente un nuevo evento tromboembólico y el cuadro clínico cambie a aquel similar al de la TEP masiva.

La TEP masiva es producida por la súbita obstrucción de más del $50 \%$ del lecho vascular por un trombo en dos o más arterias lobares, ${ }^{20}$ lo que asociado a mecanismos de vasoconstricción, se incrementa de manera significativa la presión de la arteria pulmonar impidiendo que el VD desarrolle mecanismos adaptativos suficientes y éste claudique por una sobrecarga de presión. ${ }^{11}$ A dicho estado se le conoce también como cor pulmonale agudo por su origen en la vasculatura pulmonar. La PAPm necesaria para llevar a una falla aguda del VD, a diferencia de la HP crónica, es de $40 \mathrm{mmHg}-50 \mathrm{mmHg}$. Es de notar que en la condición de falla ventricular, el nivel de la PAPm no está tan elevada, debido a la incapacidad del VD para generar mayor presión y por el bajo gasto, donde la isquemia ventricular es el factor más importante para el inicio y mantenimiento de la falla ventricular. ${ }^{25}$ Aquí, la sintomatología está relacionada con la disfunción del VD más que a la HP. La presión ventricular al final de la diástole elevada es transmitida a la aurícula derecha y la dilatación de la delgada pared ventricular ocasiona una insuficiencia tricuspídea comprometiendo el llenado ventricular y, por consecuencia, el gasto cardíaco. La dilatación del VD desplaza el tabique interventricular y junto con el retardo sistólico afectan adicionalmente el gasto cardíaco izquierdo. ${ }^{22}$ Lo anterior ocasiona el síndrome de colapso circulatorio ${ }^{26}$ manifestado por una serie de síntomas como disnea (sin ortopnea), taquicardia, hipotensión, confusión o síncope, hipoxemia, cianosis central y periférica, así como dolor torácico tipo angina secundario a isquemia ventricular debido al compromiso de la perfusión coronaria derecha. El paciente suele lucir pálido o cianótico, diaforético y taquipneico. La ingurgitación yugular es difícil de valorar por la presencia de insuficiencia respiratoria y actividad de los músculos accesorios de la respiración, pero de apreciarse ésta, el pulso yugular visible en el cuello suele relacionarse con $\mathrm{S}_{2}$ (onda « $\mathrm{V} \gg$ v venosa yugular) debido a insuficiencia tricuspídea y falla ventricular severas, y contrasta con el pulso yugular visible y más cercano al primer ruido cardíaco $\left(\mathrm{S}_{1}\right)$ de sujetos normales (onda «A» venosa yugular). ${ }^{20}$
La taquicardia hace difícil auscultar el ritmo de galope y el desdoblamiento amplio de $\mathrm{S}_{2}$. Ya puede ser auscultado un soplo sistólico en el foco tricuspídeo producido por insuficiencia tricuspídea y, debido a que el llenado ventricular derecho puede estar limitado durante la inspiración, el soplo quizá no se intensifique durante la respiración profunda, como normalmente ocurriría en otras formas de insuficiencia tricuspídea (signo de Rivero-Carvallo negativo). ${ }^{27}$ Puede haber reforzamiento del componente pulmonar de $\mathrm{S}_{2}$; $\sin$ embargo, éste no es un signo confiable ya que el flujo sanguíneo pulmonar puede estar disminuido. En pacientes con cor pulmonale agudo secundario a embolismo pulmonar, un tercer ruido $\left(\mathrm{S}_{3}\right)$ es auscultable con la campana del estetoscopio por debajo del proceso xifoideo con el paciente en posición supina e indica una falla ventricular derecha. En relación a $\mathrm{S}_{2}$, un $\mathrm{S}_{3}$ es de tonalidad más baja, y como ruido protodiastólico éste se encuentra un poco más distanciado de $\mathrm{S}_{2}$. Asimismo, un $\mathrm{S}_{3}$ derecho deberá distinguirse del $\mathrm{S}_{3}$ fisiológico, el cual por lo regular es audible en varones menores de 40 años y en mujeres menores de 45 años, y que, como con el $S_{3}$ originado por patología valvular y ventricular izquierda, son mejor auscultables en el ápice cardíaco. La presencia de un $\mathrm{S}_{3}$ derecho no es constante con cada latido cardíaco pero su intensidad, como sucede con otros eventos auscultatorios derechos, puede incrementarse también durante la inspiración debido al incremento en el flujo de sangre hacia el VD (signo de Rivero-Carvallo positivo). La dilatación del VD también puede producir un daño al sistema de conducción y un bloqueo incompleto o completo de la rama derecha del haz de His y con ello modificar algunos aspectos de la auscultación cardíaca, de forma específica un desdoblamiento de $\mathrm{S}_{1}$ o acrecentar el desdoblamiento de $\mathrm{S}_{2}{ }^{20}{ }^{2}$ La desincronización de los ventrículos puede verse exacerbada por el desarrollo del bloqueo de rama derecha. Como resultado, el llenado del ventrículo izquierdo se ve impedido en la diástole temprana, lo que puede llevar a una reducción del gasto cardíaco y contribuir a la hipotensión sistémica y la inestabilidad hemodinámica. ${ }^{22}$ No obstante todo lo anterior, existe un número significativo de pacientes que pudieran no presentar síntomas, fenómeno conocido como TEP silenciosa, lo que debe alertar al clínico en la búsqueda intencionada de otros signos sugestivos de TEP en todo paciente portador de TVP proximal a la vena poplítea. ${ }^{28}$

Analizado todo lo anterior, es importante mencionar que el cuadro clínico de TEP puede variar también según la presencia de enfermedades cardiopulmonares. Los tres síndromes comentados, descritos por primera vez por Sharma y Sasahara en $1979,{ }^{29}$ se encuentran en el 95\% de los casos documentados de TEP; empero, se vuelven aún más inespecíficos ante la presencia de EPOC o insuficiencia cardíaca congestiva y no es infrecuente no llegar al diagnóstico correcto. ${ }^{30}$ 


\section{Hipertensión pulmonar crónica}

La HP crónica abarca un grupo de varias entidades clínicas, en las que la remodelación obstructiva y la pérdida del lecho vascular pulmonar son responsables del aumento de PAPm y de la RVP, produciendo con el tiempo insuficiencia cardíaca derecha progresiva, deterioro funcional y una muerte prematura. ${ }^{31}$

Antes la HP se dividía en categorías primarias y secundarias. La HP primaria, hoy conocida como hipertensión arterial pulmonar (HAP), describía una vasculopatía hipertensiva idiopática, que afectaba exclusivamente la circulación pulmonar, mientras que la HP secundaria se asociaba con un proceso producido por una enfermedad subyacente. El diagnóstico de HAP era de exclusión, después de descartar todas las causas de HP. La reciente identificación de un gen responsable de las formas hereditarias de esta enfermedad, junto con el desarrollo de tratamientos médicos específicos y el refinamiento de las técnicas quirúrgicas, ha llevado a una clasificación revisada de HP. Durante el sexto simposio mundial de HP más reciente celebrado en Niza, Francia en el $2018,{ }^{2}$ la clasificación previa de la HP de la OMS fue mejorada con la intensión de ver reflejadas las vías genéticas, fisiopatológicas y los algoritmos de tratamiento. La HP aún se clasifica en cinco categorías diferentes, de las cuales la HAP (antes denominada HP precapilar en referencia a la ubicación del origen patológico en el sistema arterial pulmonar) está entre estos cinco grupos (grupo 1 de la OMS) y que, además incluye a la HAP hereditaria, la inducida por drogas, $\mathrm{VIH}$, hipertensión portal, entre otras causas como son aquellos respondedores a bloqueadores de los canales del calcio, enfermedad venooclusiva pulmonar y la hemangiomatosis capilar pulmonar. Los otros cuatro grupos son: HP causada por enfermedad cardíaca izquierda, incluidas las cardiopatías congénitas (grupo 2 de la OMS; a veces denominada HP «poscapilar» o hipertensión venosa pulmonar); HP debida a enfermedad pulmonar o hipoxemia (grupo 3 de la OMS); HP secundaria a enfermedad tromboembólica crónica (grupo 4 de la OMS) y diversas entidades de HP con mecanismos fisiopatológicos poco claros o multifactoriales (grupo 5 de la OMS).

El diagnóstico inadecuado, incompleto y tardío de HP no es infrecuente y se informa hasta en el $85 \%$ de los pacientes con factores de riesgo y con un retraso diagnóstico de 1 a 2 años en promedio desde la aparición de los primeros síntomas. ${ }^{32,33}$ En la TEP crónica se describen períodos mucho mayores para manifestar algún síntoma, período conocido como de «luna de miel». ${ }^{34}$ Específicamente, los pacientes son evaluados en la búsqueda de cualquier antecedente familiar de HAP o una historia o evidencia de enfermedad cardíaca congénita, enfermedades autoinmunes, enfermedad hepática, enfermedad tromboembólica, historia de uso de estimulantes, enfermedad pulmonar subyacente, cardiopatía isquémica, valvular o hipertensiva, desórdenes respiratorios del sueño e infección por $\mathrm{VIH}$. El grupo etario tiene especial importancia. La HAP suele afectar a mujeres relativamente jóvenes, mientras que en los adultos mayores el síndrome coronario, la cardiopatía isquémica o hipertensiva, fibrilación auricular, cor pulmonale crónico e hipotiroidismo se presentan con mayor frecuencia como causas de HP.

La circulación pulmonar normal es única en su capacidad para acomodar todo el gasto cardíaco manteniendo una relativa baja presión arterial pulmonar, incluso durante un ejercicio máximo. Esto se logra a través de su circuito de alta capacitancia y baja resistencia, con grandes reservas de microcirculación que no son reclutadas durante el reposo. Durante el ejercicio, la microcirculación pulmonar es reclutada de manera progresiva, lo que resulta en el mantenimiento de una presión arterial relativamente baja a pesar de un flujo sanguíneo pulmonar creciente. El reclutamiento de la microcirculación también sirve para aumentar el área de superficie capilar disponible para el intercambio de gases durante el ejercicio. La alta capacitancia de la circulación pulmonar significa que en etapas tempranas de una HP crónica pueda compensarse bien. De hecho, el 50\% de la circulación pulmonar debe estar obstruida antes de que se detecte un aumento en la PAPm en reposo.

La HAP es una enfermedad clínicamente silenciosa hasta que la presión de la arteria pulmonar aumenta relativamente tarde en su historia natural, sólo cuando más del $60 \%$ de las arterias pulmonares distales pequeñas se han obstruido. La sintomatología y los hallazgos de la exploración física dependerán de la gravedad del trastorno en la vasculatura pulmonar y del grado de disfunción ventricular derecha. Aunque subjetiva, la clase funcional de la New York Heart Association (NYHA)/OMS proporciona una evaluación importante de los síntomas y la capacidad funcional asociada con los resultados en muchos estudios. Al inicio, conforme incrementa paulatinamente la PAPm, la plasticidad normal del lecho vascular pulmonar y los mecanismos normales para acomodar el flujo sanguíneo extra generado en situaciones donde se incrementa el gasto cardíaco se van perdiendo, por lo que los mecanismos para optimizar un desequilibrio entre la ventilación-perfusión por medio del reclutamiento y distensión de los vasos pulmonares, no son suficientes. Los síntomas iniciales en la HP crónica aparecen sólo después de una adaptación fallida del VD al aumento de la poscarga y no son específicos. ${ }^{35}$ Tomando como prototipo de HP a la forma primaria, los pacientes refieren múltiples síntomas inespecíficos incluyendo disnea, fatiga, malestar torácico, palpitaciones, junto con una limitación del ejercicio a partir de una presión arterial pulmonar sistólica (PAPs) aproximada a los $50 \mathrm{mmHg}$. La fatiga es un síntoma multidimensional definido como una sensación de agotamiento abrumadora, debilitante 
y sostenida que disminuye la capacidad de la persona de llevar a cabo las actividades diarias para trabajar con eficacia. ${ }^{36}$ La fatiga contiene componentes físicos, cognitivos y afectivos sobrepuestos. En el aspecto físico, los pacientes sienten que no tienen la energía para realizar actividades. Cognitivamente, los pacientes pueden tener dificultades para concentrarse y pueden carecer de motivación para participar en las actividades cotidianas. Más del $90 \%$ de los pacientes con HAP reportan fatiga que interfiere con su calidad de vida. ${ }^{36}$ No es referida comúnmente por los pacientes, sino hasta que ésta se asocia con disnea y junto con la angustia o ansiedad pueden interferir con la función cognitiva, causar depresión, pérdida de memoria y afectar negativamente el estado funcional en la HAP. En más de una tercera parte de estos pacientes cursan con algún desorden psiquiátrico asociado y hasta un $24 \%$ de ellos pueden haber recibido algún tipo de tratamiento. ${ }^{37}$ La fatiga como la disnea, se cree que están relacionadas con la incapacidad de un corazón derecho crónicamente hipertensivo para aumentar de manera adecuada el gasto cardíaco en situaciones de estrés, así como a la hipoxemia causada por la subóptima relación ventilación-perfusión. Los pacientes con esclerosis sistémica u otras enfermedades del tejido conectivo tienen causas multifactoriales que contribuyen a la intolerancia al esfuerzo, incluidas las afecciones musculoesqueléticas..$^{38} \mathrm{La}$ disnea presentada en los casos de HAP no se presenta en forma de ortopnea ni en disnea paroxística nocturna. Su inicio no es súbito y no suele fluctuar durante el día ni se asocia a pérdida del ritmo sueño-vigilia, síntomas que pudieran estar en su mayoría asociados a un componente psicosomático. Por otro lado, aquellos pacientes con enfermedades respiratorias crónicas, que cursen con una disnea en grado desproporcional a su mecánica respiratoria o intercambio gaseoso, deberán ser considerados para escrutinio de HP.

Algunos síntomas específicos a ciertas enfermedades de base también son con frecuencia referidos, por ejemplo fenómeno de Raynaud en las colagenopatías o somnolencia diurna en los casos de apnea obstructiva del sueño.

El hecho de que la sobrecarga de presión se instale de manera progresiva permite que ocurran mecanismos de compensación adaptativa como lo es la hipertrofia, y con la finalidad de mantener en el tiempo su función. De esta manera, la remodelación del VD puede sostener la función ventricular por largos períodos de tiempo y contribuir a la circulación sistémica de manera adecuada. En pacientes que tienen un alto riesgo de padecer una HAP crónica o se sospecha que ya la tienen con base en los síntomas comentados, se debe prestar atención especial a los signos físicos tempranos que incluyen inicialmente el reforzamiento del componente pulmonar de $\mathrm{S}_{2}$ y un desdoblamiento estrecho de $S_{2}$ que aumentan un poco con la inspiración profunda, pero conforme incremente con el tiempo la PAPm, este des- doblamiento se volverá fijo; es decir, con menos variación respiratoria y más si hubiera un bloqueo de rama derecha del haz de His producida por fibrosis endomiocárdica que afectará al sistema de conducción. ${ }^{39}$ Recuérdese que el desdoblamiento fijo y amplio de $\mathrm{S}_{2}$ también es característico de la comunicación interauricular (CIA).

El componente pulmonar de $\mathrm{S}_{2}\left(\mathrm{~S}_{2 \mathrm{p}}\right)$ debe ser tan fuerte como el aórtico $\left(\mathrm{S}_{2 \mathrm{a}}\right)$ en el foco pulmonar durante la inspiración en un paciente con presiones normales de la arteria pulmonar. A medida que aumenta la PAPs, la intensidad de $\mathrm{S}_{2 \mathrm{p}}$ debería aumentar con respecto a $\mathrm{S}_{2 \mathrm{a}}$. Según Bourge, ${ }^{40}$ la intensidad relativa de $S_{2 p}$ igual o mayor que $S_{2 a^{\prime}}$, se ausculta más lejos del foco pulmonar a medida que aumenta la PAPs. La intensidad de $S_{2 p}$ en comparación con $S_{2 a}$ se evalúa auscultando a lo largo del borde esternal izquierdo, comenzando en el segundo espacio intercostal izquierdo (foco pulmonar), luego los espacios intercostales paraesternales tercero, cuarto y quinto, continuar con el quinto espacio en un punto entre el borde esternal izquierdo y la línea medioclavicular y enseguida el quinto espacio intercostal en la línea medioclavicular (figura 2). La intensidad relativa de $S_{2 p}$ sobre $S_{2 a}$ se evalúa en cada área y la presión PAPs se calcula en función de donde $S_{2 p}$ es igual o más alto que $\mathrm{S}_{2 \mathrm{a}}$ de la siguiente forma:

\begin{tabular}{|l|l|l|}
\hline a) & $\begin{array}{l}2^{\circ} \text { espacio intercostal paraesternal izquierdo } \\
\text { (foco pulmonar) }\end{array}$ & $25-35 \mathrm{mmHg}$ \\
\hline b) & $3^{\circ}$ espacio intercostal paraesternal izquierdo & $35-45 \mathrm{mmHg}$ \\
\hline c) & $4^{\circ}$ espacio intercostal paraesternal izquierdo & $45-55 \mathrm{mmHg}$ \\
\hline d) & $5^{\circ}$ espacio intercostal paraesternal izquierdo & $55-65 \mathrm{mmHg}$ \\
\hline e) & $\begin{array}{l}5^{\circ} \text { espacio intercostal izquierdo entre el } \\
\text { borde esternal y la línea medioclavicular }\end{array}$ & $65-75 \mathrm{mmHg}$ \\
\hline f) & $\begin{array}{l}5^{\circ} \text { espacio intercostal izquierdo a nivel de la } \\
\text { línea medioclavicular }\end{array}$ & $>75 \mathrm{mmHg}$ \\
\hline
\end{tabular}

Estos hallazgos recién fueron corroborados por Mcnab y colaboradores al ser comparados con medidas obtenidas mediante ecocardiografía Doppler. ${ }^{41}$ Bourge describe como limitantes de su método para estimar la PAPs, la incapacidad para caracterizar de manera correcta el desdoblamiento de $\mathrm{S}_{2}$ impidiendo así la estimación de la PAPs; también en individuos obesos, en casos de estenosis mitral o presencia de una válvula aórtica o pulmonar protésica, en EPOC grave o con enfermedad venooclusiva pulmonar. Si una enfermedad pulmonar es la causa de la HP, la presión de la arteria pulmonar no reflejará con precisión las presiones de llenado del ventrículo izquierdo. ${ }^{40}$

Con el VD aún hipertrofiado y con una PAPm considerablemente elevada podría observarse una onda «A» yugular prominente, levantamiento sistólico del segundo espacio intercostal izquierdo, un movimiento oscilante 


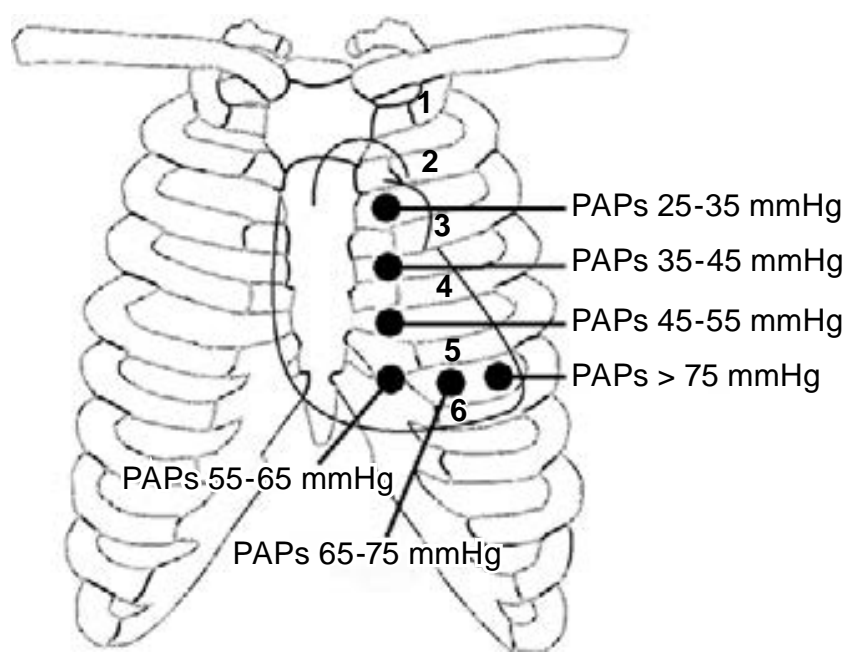

Figura 2: Puntos auscultatorios para estimar la presión arterial pulmonar sistólica (PAPs) por el método de Bourge.

Modificado de Leier CV et al. ${ }^{40}$

esternal que combinado con la retracción simultánea del ápice cardíaco produce una apariencia de «balanceo», un choque de cierre de la pulmonar palpable, matidez mayor a $2.5 \mathrm{~cm}$ por fuera del borde paraesternal izquierdo a nivel del segundo espacio intercostal y un levantamiento palpable (con el talón de la mano) paraesternal bajo izquierdo que corresponde al área de proyección del VD (complejo de la pulmonar de Chávez). La auscultación percutoria o pleximétrica pudiera ser de ayuda en delimitar la silueta cardíaca colocando el estetoscopio en la parte baja del esternón y percutiendo de forma simultánea con el dedo medio desde el borde cardíaco izquierdo medialmente hacia el estetoscopio (de afuera hacia adentro). ${ }^{42}$ El borde cardíaco izquierdo es determinado percutiendo desde arriba en el área del hilio. Sin mover el estetoscopio se hace lo mismo con el borde cardíaco derecho. A la par se puede percutir desde el estetoscopio hacia afuera localizando los mismos márgenes cardíacos al disminuir la intensidad de la nota de percusión (de adentro hacia afuera). ${ }^{42} \mathrm{~A}$ la auscultación cardíaca con el diafragma pudiera encontrarse un clic o chasquido de eyección sistólica temprana, el cual deberá distinguirse del desdoblamiento de $\mathrm{S}_{1}$ por tener un tono e intensidad más elevado, se ausculta mejor en el foco pulmonar más que en el tricuspídeo y es ocasionado por la resistencia de la válvula pulmonar a su apertura durante el inicio de la sístole ventricular derecha. Es el único sonido patológico originado en el lado derecho del corazón que incrementa en intensidad durante la espiración y disminuye o desaparece durante la inspiración, siempre y cuando el anillo valvular no se encuentre significativamente dilatado. ${ }^{22,43}$ Esta característica lo distingue del desdoblamiento de $\mathrm{S}_{1}$ el cual se ausculta mejor en el foco tricuspídeo y no varía con la respiración. La rigidez del VD hipertrofiado puede hacer evidente un $\mathrm{S}_{4}$ derecho inspiratorio y con una irradiación a las venas yugulares del cuello. También podrá auscultarse un soplo eyectivo pulmonar mesosistólico en el borde esternal izquierdo que incrementa con la maniobra de Rivero-Carvallo y que no deberá ser confundido con el soplito inocente del tracto de salida del VD auscultado en sujetos normales a cualquier edad, en la anemia o en la tirotoxicosis, pero sobre todo en aquéllos con pectus excavatum y en el síndrome de espalda recta, donde el soplo se produce a causa de la compresión del tracto de salida del ventrículo derecho por el esternón y desaparece con la inspiración profunda. ${ }^{44}$ La válvula tricúspide es una válvula débil con tendencia fácil a las «fugas» y la presencia del soplo de reflujo tricuspídeo junto con la presencia de ondas « $\mathrm{V} »$ no necesariamente indican una falla del ventrículo derecho. ${ }^{1}$ Cuando aún la PAPm no es muy elevada y la presión dentro del VD es normal, puede auscultarse un soplo sistólico temprano de insuficiencia tricuspídea debido a que las presiones de la aurícula y del VD se equilibran a mitad de la sístole, eliminando así el gradiente causante del soplo. ${ }^{20}$

Los síntomas presentados en una HAP crónica avanzada están relacionados con la dilatación e isquemia del VD. EI dolor torácico puede tener las características de una angina típica o atípica y en principio ser causado por el incremento en la demanda de oxígeno del VD y después pudiera ser causada por la compresión de la arteria coronaria izquierda por la arteria pulmonar dilatada significativamente (diámetro del tronco de la arteria pulmonar $>40 \mathrm{~mm}$ ). La dilatación de la arteria pulmonar también pudiera comprimir el nervio laríngeo recurrente y ocasionar disfonía (síndrome cardiovocal de Ortner). ${ }^{45}$ Otros síntomas menos frecuentes son la tos y la hemoptisis. Esta última, aunque rara en la HAP, suele ser común en el síndrome de Eisenmenger, enfermedad tromboembólica crónica, enfermedad de Rendu-Osler, vasculitis, y en las anomalías arteriales pulmonares. El bajo gasto del VD y el desplazamiento del tabique interventricular por la dilatación maladaptativa del VD podrían afectar el gasto cardíaco izquierdo y manifestarse por cuadros de lipotimias, síncope o crisis convulsivas.

En etapas avanzadas, la sintomatología está relacionada con el bajo gasto cardíaco, o bien con la disfunción diastólica del VD. La falla del VD puede definirse como el síndrome clínico resultante de la incapacidad del corazón derecho para proporcionar un flujo de sangre adecuado a la circulación pulmonar con una presión venosa central normal. Los síntomas producidos por bajo gasto suelen presentarse más en la HAP, básicamente cuadros de lipotimias, síncope, crisis convulsivas o inclusive la muerte súbita. ${ }^{46}$ Los síntomas congestivos por disfunción diastólica del VD se observan más en las enfermedades respiratorias crónicas, p. ej. hepatalgia, la plenitud abdominal por ascitis 
y edema de las extremidades inferiores. ${ }^{46}$ No obstante, en algunos pacientes con EPOC, la hipercapnia se asocia con un aumento en la reabsorción renal de bicarbonato, que sirve para minimizar la caída del pH arterial; pero también puede contribuir a la formación de edema, ya que promueve la reabsorción pasiva de sodio y agua. ${ }^{47}$

En esta situación, suelen observarse los signos de regurgitación valvular e insuficiencia cardíaca derecha que incluyen una presión venosa yugular elevada con ondas «V》 acentuadas y en presencia de un soplo holosistólico de regurgitación tricuspídea debido a la dilatación del anillo de la válvula tricúspide, que ocasiona la inadecuada coaptación de sus valvas y permite el reflujo de sangre hacia la aurícula derecha desde el VD durante toda la sístole. Dicho soplo se ve acentuado durante la inspiración profunda (signo de Rivero-Carvallo positivo) o durante la compresión manual sobre el hígado. El paciente no únicamente tiene que estar acostado y con la cabecera levantada a $45^{\circ}$ para observar las pulsaciones venosas, sino también éstas pueden ser evidentes en posición sentada a $90^{\circ}$. Las pulsaciones venosas yugulares internas se pueden confundir con las pulsaciones carotideas, en particular cuando hay una onda «V» prominente, como se ve por lo regular en la regurgitación tricuspídea. Esta confusión se evita por el hecho de que el pulso carotideo es fácilmente palpable y no fácilmente compresible, mientras que las pulsaciones venosas son compresibles y por lo común no son palpables. ${ }^{40}$ No obstante, estas pulsaciones venosas si pueden percibirse a la palpación en el hígado. El signo de Kussmaul es la elevación paradójica de la presión venosa central durante la inspiración. En personas normales, la presión venosa cae durante la inspiración puesto que las presiones en el lado derecho del corazón disminuyen como la presión intratorácica desciende. Este signo es típico en la pericarditis constrictiva, pero también en la insuficiencia cardíaca severa, TEP y otras causas de falla del VD como lo es la isquemia y la sobrecarga de volumen.

El soplo diastólico de regurgitación pulmonar (de Graham Steell) es localizado sobre el foco pulmonar, de tono elevado y muy breve en duración, suele ser precedido por un $\mathrm{S}_{2}$ intenso y aumentar de manera considerable con la inspiración. Aun así, con el tiempo la disminución del gasto cardíaco derecho puede aminorar la intensidad de $\mathrm{S}_{2}$ La dilatación ventricular derecha produce una disminución de la distensibilidad de sus paredes y conducir a un $S_{3}$ derecho que al igual que un $\mathrm{S}_{4^{\prime}}$ es más evidente durante la inspiración profunda. Es interesante como los pacientes con $\mathrm{HAP}$, incluso con formas graves de la enfermedad, tienden a permanecer en ritmo sinusal, a diferencia de aquellos casos de HP secundaria a insuficiencia cardíaca izquierda.

Algunos hallazgos al examen físico también pudieran orientarnos hacia la etiología específica de la HP, p.ej. la flegmasia alba o cerúlea dolens de la TVP de las extremidades inferiores, la redundancia del paladar blando en casos de apnea obstructiva del sueño, la candidiasis oral del paciente con sida, la bradicardia y el mixedema en el hipotiroidismo, el tórax en tonel del paciente con EPOC, la cifoscoliosis en pacientes con síndrome de insuficiencia torácica, los signos de Silvestrini-Corda de la cirrosis hepática, los cambios cutáneos o artritis característica de algunas colagenopatías, y el hipocratismo digital del paciente con enfermedad pulmonar intersticial difusa fibrosante, cardiopatías congénitas o enfermedad venooclusiva pulmonar.

La cianosis es común en pacientes con cardiopatía congénita y HP. La derivación de derecha a izquierda reduce el contenido de oxígeno arterial y se asocia con una elevada producción renal de eritropoyetina, promoviendo así la eritropoyesis y la eritrocitosis secundaria. Los niveles elevados de hemoglobina representan una adaptación fisiológica a la cianosis crónica y es esencial para mantener una adecuada oxigenación tisular y prevenir el daño hipóxico del órgano terminal. Los niveles elevados de hematócrito se han relacionado con síntomas de hiperviscosidad como cefaleas, mareos, trastornos visuales, parestesias y mialgias, aunque existen datos sobre la asociación entre el hematocrito y la viscosidad, no es concluyente y se confunde con la deficiencia de hierro. Los soplos debidos a cortocircuitos previos de izquierda a derecha a nivel ventricular (sistólico) o arterial (soplo continuo) por lo común desaparecen tan pronto como se desarrolla la fisiología de Eisenmenger. ${ }^{48} \mathrm{~A}$ diferencia de otras formas de $\mathrm{HP}, \mathrm{S}_{2 \mathrm{a}}$ y $\mathrm{S}_{2 \mathrm{p}}$ se fusionan sin presentar un desdoblamiento durante la inspiración escuchándose así a la auscultación como un $\mathrm{S}_{2}$ «único» y es debido a la disminución de la capacitancia del lecho vascular pulmonar y la disfunción del VD. Las arritmias son frecuentes secuelas tardías en pacientes con fisiología de Eisenmenger y pueden allanar el camino para el deterioro clínico, la insuficiencia cardíaca o la muerte súbita cardíaca.

\section{CONCLUSIÓN}

Aceptando las limitaciones inherentes a las habilidades de cada clínico y a que la evidencia científica es muy limitada en evaluar el valor diagnóstico de algunos signos físicos, al no contar con estudios con el número suficiente de pacientes para ser aplicables en todos los grupos de población; además de que el diagnóstico de una enfermedad que rara vez depende de la presencia de un signo o síntoma y de que muchas veces los investigadores no son clínicos con el tiempo suficiente para estar al lado del paciente; y de que el tiempo requerido para realizar un verdadero examen físico va en contra de las prácticas actuales de atención médica colectiva y de la gran carga laboral del médico; todo esto hace aún de la clínica un método diagnóstico básico indispensable.

Mejor aún, por su economía, portabilidad, repetitividad, no invasividad e inocuidad, sobre todo en países 
subdesarrollados como el nuestro, en donde no es posible contar en todas las unidades médicas con equipo médico sofisticado, sobre todo departamentos de hemodinamia. La ecocardiografía, considerada un subrogado de gran utilidad, no deja de ser un estudio operador dependiente que no puede estimar la presión de la arteria pulmonar en un $10 \%-25 \%$ de los pacientes, presenta una variabilidad entre un estudio a otro y la correlación con la hemodinámica pulmonar algunas veces no es consistente.

\section{REFERENCIAS}

1. Peacock AJ. The diagnosis of pulmonary hypertension. Clinical features. In: Peacock AJ, Rubin LJ, editors. Pulmonary circulation. Diseases and their treatment. $2^{\mathrm{a}}$ ed. New York: Arnold publishers; 2004.p.73.

2. Galiè N, McLaughlin VV, Rubin LJ, Simonneau G. An overview of the 6th World Symposium on Pulmonary Hypertension. Eur Respir J 2019;53(1).pii1802148. https://doi.org/10.1183/13993003.02148-2018.

3. Guadalajara-Boo JF. La auscultación del corazón, un arte en vías de extinción. Gac Med Mex 2015;151(2):260-265.

4. Patil S, Henry JW, Rubenfire M, Stein PD. Neural network in the clinical diagnosis of acute pulmonary embolism. Chest 1993;104(6):16851689. https://doi.org/10.1378/chest.104.6.1685.

5. Freeman LM, Krynyckyi B, Zuckier LS. Enhanced lung scan diagnosis of pulmonary embolism with the use of ancillary scintigraphicfinfdings and clinical correlation. Semin Nucl Med 2001;31(2):143-157.

6. Colman R, Whittingham H, Tomlinson G, Granton J. Utility of the physical examination in detecting pulmonary hypertension. A mixed methods study. PLoSOne 2014;9(10):e108499. https://doi.org/10.1371/ journal.pone.0108499.

7. Marcus GM, Vessey J, Jordan MV, et al. Relationship between accurate auscultation of a clinically useful third heart sound and level of experience. Arch Intern Med 2006;166(6):617-622. https:// doi.org/10.1001/archinte.166.6.617.

8. Flores-Franco RA. La auscultación como metodología diagnóstica. En: Flores-Franco RA, Gómez-Sáenz LM, editores. La estetoscopia. Teoría, práctica y enseñanza-aprendizaje. México: Trillas; 2018.p.29.

9. Granton JT, Whittingham H, Tomlinson $\mathrm{G}$, et al. Does this patient have pulmonary hypertension? A prospective evaluation of the utility of the physical examination. Am J Respir Crit Care Med 2010;181:A3372. https://doi.org/10.1164/ajrccmconference.2010.181.1_MeetingAbstracts.A3372-

10. Fraser AG, Weston CF. The Graham Steell murmur: eponymous serendipity? J R Coll Physicians Lond 1991;25(1):66-70.

11. Hui-Li G. The management of acute pulmonary arterial hypertension. CardiovascTher 2011;29(3):153-175. https://doi.org/10.1111/j.17555922.2009.00095.x.

12. Wells PS, Anderson DR, Rodger M, et al. Derivation of a simple clinical model to categorize patients probability of pulmonary embolism: Increasing the models utility with the SimpliRED D-dimer. Thromb Haemost 2000;83(3):416-420.

13. Stein PD. Relation of right-sided pressures to clinical characteristics of patients with no prior cardiopulmonary disease. In: Stein PD, editor. Pulmonary embolism. 3rd ed. Oxford, UK: Wiley Blackwell; 2016.p. 272-274.

14. Stein PD, Beemath A, Matta F, et al. Clinical characteristics of patients with acute pulmonary embolism: data from PIOPED II. Am J Med 2007;120(10):871-879.
15. Light RW. Pleural effusion due to pulmonary emboli. CurrOpinPulm Med2001;7(4):198-201.

16. Stein PD. Clinical characteristics of patients with acute pulmonary embolism stratified according to their presenting syndromes. In: Stein PD, editor.Pulmonary embolism. 3rd ed. Oxford, UK: Wiley Blackwell; 2016.p. 280-285.

17. Riedel M. Diagnosing pulmonary embolism. Postgrad Med J 2004;80(944):309-319.

18. Lobo-Beristáin JL, Egea-Santaolalla C. Síndromes clínicos de embolia pulmonar. Semin Fund Esp Reumatol 2006;7(3):139-146. doi: 10.1016/S1577-3566(06)75092-8.

19. Hughes MJ, Stein PD, Matta F. Silent pulmonary embolism in patients with distal deep venous thrombosis: systemic review. Thromb Res 2014;134(6):1182-1185. https://doi.org/10.1016/j.thromres.2014.09.036.

20. Hill NS. The cardiac exam in lung disease. Clin Chest Med 1987;8(2):273-285.

21. Don Michael TA. Eliciting heart sounds: timing, amplitude, and pitch. In: Don Michel TA, editor. Auscultation of the heart. A cardiophonetic approach. New York, USA: McGraw-Hill; 1998.p.113-160.

22. Mauritz GJ, Marcus JT, Westerhof N, Postmus PE, Vonk-Noordegraaf A. Prolonged right ventricular post-systolic isovolumic period in pulmonary arterial hypertension is not a reflection of diastolic dysfunction. Heart 2011;97(6):473-478. https://doi.org/10.1136/ hrt.2010.193375.

23. Fraser RS, Lynne-Davies P. Continuous chest murmur acquired following pulmonary thromboembolism. Chest 1974;65:562-564.

24. ZuWallack RL, Liss JP, Lahiri B. Acquired continuous murmur associated with acute pulmonary thromboembolism. Chest 1976;70(4):557-559. https://doi.org/10.1378/chest.70.4.557.

25. Santos-Martínez LE. Fisiopatología de la falla ventricular derecha en la hipertensión arterial pulmonar. Arch Cardiol Mex 2004;74(Supl 2):S353-S357.

26. Renshaw SA, Brennan SR, Heigenbottam TW. The clinical presentation of deep vein thrombosis and pulmonary thromboembolism. In: Oudkerk M, van Beek EJR, ten Cate JW, editors. Pulmonary embolism. Oxford, UK: Blackwell Science; 1999.p.34-42.

27. Shank-Coviello J. Fundamentos de los soplos. En Shank-Coviello J, editor. Manual interactivo de auscultacióncardíaca y respiratoria. $5^{\text {a }}$ ed. Philadelphia, PA: Wolters Kluwer/Lippincott Williams \& Wilkins; 2016.p.56-78.

28. Stein PD, Matta F, Musani MH, Diaczok B. Silent pulmonary embolism in patients with deep venous thrombosis: a systemic review. Am J Med 2010;123(5):426-431. https://doi.org/10.1016/j. amjmed.2009.09.037.

29. Sharma GV, Sasahara AA. Diagnosis and treatment of pulmonary embolism. Med Clin North Am 1979;63(1):239-250.

30. Manganelli D, Palla A, Donnamaria V, Giuntini C.Clinical features of pulmonary embolism. Doubts and certainties. Chest 1995;107(1 Suppl):25S-32S. https://doi.org/10.1378/chest.107.1_ supplement.25s.

31. Simonneau G, Galiè N, Rubin L, et al. Clinical classification of pulmonary hypertension. J Am Coll Cardiol 2004;43(12 Suppl):S5-S12.

32. Maron BA, Choudhary G, Kahn UA, et al. The clinical profile and underdiagnosis of pulmonary hypertension in US veteran patients. CircHeart Fail 2013;6(5):906-912. https://doi.org/10.1161/ CIRCHEARTFAILURE.112.000091.

33. Deaño RC, Glassner-Kolmin C, Rubenfire M, et al. Referral of patients with pulmonary hypertension diagnoses to tertiary pulmonary hypertension centers: the multicenter RePHerral study. 
JAMA Intern Med 2013;173(10):887-893. https://doi.org/10.1001/ jamainternmed.2013.319.

34. Humbert M. Pulmonary arterial hypertension and chronic thromboembolic pulmonary hypertension: pathophysiology. Eur Respir Rev 2010;19(115): 59-63. https://doi.org/10.1183/09059180.00007309.

35. Lai YC, Potoka KC, Champion HC, Mora AL, Gladwin MT. Pulmonary arterial hypertension: the clinical syndrome.Circ Res 2014;115(1):115130. https://doi.org/10.1161/CIRCRESAHA.115.301146.

36. Matura LA, Shou H, Fritz JS, et al. Physical activity and symptoms in pulmonary arterial hypertension. Chest 2016;150(1):46-56.https:// doi.org/10.1016/j.chest.2016.02.633

37. Delcroix M, Howard L. Pulmonary arterial hypertension: the burden of disease and impact on quality of life. Eur Respir Rev 2015;24:621-629.

38. Dumitrescu D, Sitbon O, Weatherald J, Howard LS. Exertional dyspnoea in pulmonary arterial hypertension. Eur Respir Rev 2017;26(145).pii:170039. https://doi.org/10.1183/16000617.0039-2017.

39. Sun PY, Jiang X, Gomberg-Maitland M, et al. Prolonged QRS duration: a new predictor of adverse outcome in idiopathic pulmonary arterial hypertension. Chest 2012;141(2):374-380. https://doi.org/10.1378/ chest.10-3331.

40. Leier CV,Young JB, Levine TB, et al. Nuggets, pearls, and vignettes of master heart failure clinicians. Part 2-the physical examination. Congest Heart Fail 2001;7(6):297-308.

41. Mcnab P, Castro P, Verdejo H, Martínez G. Estimación auscultatoria de la presión sistólica de arteria pulmonar: ¿Es factible? Correlación con determinación ecocardiográfica. Rev Med Chile 2010;138(11):13511356.

42. Brunk SF. Auscultatory percussion: an added dimension in physical diagnosis. Int J Clin Pract 2003;57(3):209-209.

43. Caplin M, Moshier AL. Abnormal heart sounds: other diastolic and systolic sounds.In: Caplin M, Moshier AL, editors. Auscultation Sskills.Breath \& heart sounds. $3^{\text {ra }}$ ed. Ambler, PA:Williams\& Wilkins; 2006.p.53.

44. Gómez-Sáenz LM. Auscultación cardíaca. En: Flores-Franco RA, Gómez-Sáenz LM, editores. La estetoscopia. Teoría, práctica y enseñanza-aprendizaje. México: Trillas; 2018.p.80.

45. Mandel J. Approach to the patient with pulmonary hypertension. In: Mandel T, editor. Pulmonary vascular disease. Philadelphia, PA: Saunders Elsevier; 2006.p.84-85.

46. Sandoval-Zárate J. Right ventricle insufficiency in pulmonary arterial hypertension. Physiopathologic considerations. Arch Cardiol Mex 2001;71 Suppl1:S164-S167.

47. Bhattacharya A. Cor pulmonale. JIACM 2004;5(2):128-136.

48. Diller GD, Gatzoulis MA. Pulmonary vascular disease in adults with congenital heart disease. Circulation 2007;115(8):1039-1050. https:// doi.org/10.1161/CIRCULATIONAHA.105.592386.

Conflicto de intereses: Los autores declaran no tener ningún conflicto de intereses. 